\title{
Razones para co-destruir en plataformas hoteleras usando boca a boca electrónico (e-WOM) negativo
}

\author{
Catalina Figueroa-Fadic ${ }^{1}$, Consuelo Tello-Álvarez ${ }^{1}$, Iván Veas-González ${ }^{2}$ y Cristian L. Vidal-Silva ${ }^{2 *}$ \\ (1) Ingeniería Comercial, Facultad de Economía y Administración, Universidad Católica del Norte, Angamos 0610, \\ Antofagasta - Chile (correo-e: catalina.figueroa@alumnos.ucn.cl, consuelo.tello@alumnos.ucn.cl). \\ (2) Departamento de Administración, Facultad de Economía y Administración, Universidad Católica del Norte, Angamos \\ 0610, Antofagasta - Chile (correo-e: iveas@ucn.cl, cristian.vidal@ucn.cl).
}

* Autor a quien debe ser dirigida la correspondencia

Recibido Sep. 22, 2020; Aceptado Nov. 23, 2020; Versión final Ene. 27, 2021, Publicado Jun. 2021

\begin{abstract}
Resumen
Este trabajo busca identificar motivaciones de clientes de servicios hoteleros para co-destruir el valor de estos servicios por medio del boca a boca electrónico (e-WOM, en inglés) negativo en las diferentes plataformas web. Se proponen como motivaciones la desconfirmación y desconfianza con e-WOM, además de la insatisfacción, la venta de sentimientos negativos y el altruismo. A partir de encuestas en línea se buscó identificar a usuarios que hayan tenido conductas co-destructivas hacia servicios que adquirieron; es decir, personas que hayan experimentado una situación negativa con algún proveedor de servicios hoteleros durante los últimos 12 meses y que hayan generado e-WOM negativos. Por medio de SMARTPLS, se buscó contrastar las relaciones propuestas. Los resultados del estudio muestran las motivaciones que llevan al cliente hotelero a co-destruir a través del e-WOM negativo en diferentes plataformas web, frente a experiencias negativas. Se concluye que las personas que co-destruyen por medio de e-WOM negativo realizan esta acción por la insatisfacción con el servicio recibido.
\end{abstract}

Palabras clave: co-destrucción; valor; e-WOM negativo; co-creación; lógica dominante del servicio

\section{Reasons to co-destroy hotel platforms using negative electronic Word of Mouth (e-WOM)}

\begin{abstract}
This study seeks to identify the motivations of hotel service clients to co-destroy the value of these services by negative electronic word of mouth (e-WOM) on different web platforms. Disconfirmation and distrust with eWOMs are proposed as motivations, in addition to dissatisfaction, the sale of negative feelings, and altruism. Online surveys are conducted to identify users who have had co-destructive behaviors towards services they purchased. This includes users who have experienced a hostile situation with a hotel service provider during the last 12 months and who have generated negative e-WOMs. This is examined by using SMARTPLS. The results show the motivations that lead the hotel customer to co-destroy through negative e-WOM on different web platforms in the face of negative experiences. It is concluded that users who co-destroyed by negative eWOM are motivated by dissatisfaction with the customer service received.
\end{abstract}

Keywords: co-destruction; value; negative e-WOM; co-creation; service dominant logic 


\section{INTRODUCCIÓN}

La Web proporciona un terreno fértil para la comunicación boca a boca y cada vez más consumidores escriben y comparten en línea, experiencias relacionadas con productos. Dada la naturaleza experiencial del turismo, tal conocimiento de primera mano, comunicado por otros viajeros, es especialmente útil para la toma de decisiones de viaje. Sin embargo, tal como resalta (Gonçalves et al., 2018), aún se sabe muy poco acerca de las motivaciones de los consumidores a escribir reseñas de viajes en línea. Según (Media, 2020), la Asociación Europea de Hoteles, Restaurantes y Cafés (HOTREC), junto a la Universidad de Ciencias Aplicadas de Suiza Occidental Valais demuestran que las redes sociales y las plataformas de reservas hoteleras en internet se han vuelto fundamentales al momento de planificar un viaje. Se destaca el dominio de Booking (Booking, 2020) con un 66\% del mercado y el aumento de proporción de reservas a través de las plataformas online pasando de un $23,1 \%$ a $26,1 \%$ entre el año 2015 y 2017.

Un número cada vez mayor de reservas de hoteles se realiza por medio de internet y es probable que el proceso de decisión de compra se vea influenciado por lo que publican otros huéspedes (Sparks y Browning 2010). Los comentarios o boca a boca electrónico (e-WOM) han incrementado su alcance hacia los consumidores y se han convertido en una fuente de información relevante para los mismos (Nam et al., 2018). El e-WOM en las plataformas de reservas hoteleras tales como Booking (2020) puede ser tanto positivo como negativo. Tal como indica (Nam et al., 2020), el e-WOM negativo representa la acción de emitir un comentario negativo con respecto al servicio recibido donde las expectativas que se habían formado previamente no fueron alcanzadas, lo cual resulta en una co-destrucción de valor.

El término co-destrucción hace mención al uso indebido por al menos de una de las partes, es decir, un mal uso accidental de recursos de los sistemas que interactúan y que tiene el potencial de disminuir el bienestar (Plé, 2017). La co-destrucción es la acción contraria al proceso de co-creación de valor que genera resultados positivos para los actores involucrados (Grönroos y Gummerus, 2014); y puede ocurrir como resultado de un desacuerdo entre actores, cuando existen oportunidades desiguales entre las partes involucradas durante el intercambio de recursos (Prior y Marcos-Cuevas, 2016), cuando los recursos se utilizan de manera inapropiada (Worthington y Durkin, 2012) o como resultado de la mala conducta del consumidor (Kashif y Zarkada, 2015).

Aunque se ha realizado una exhaustiva investigación respecto de las interacciones de servicio en el contexto hotelero, estas se basan principalmente en términos de co-creación de valor y experiencias positivas de servicio (Prebensen y Xie, 2017; Busser y Shulga, 2018; Casais et al., 2020; Fan et al., 2020). Existe una falta de investigación activa en las experiencias negativas que llevan a la co-destrucción de valor (Järvi et al., 2020, Ukeje et al. 2020). Dado lo anterior, el principal objetivo de este trabajo es determinar las razones o motivaciones que llevan a los usuarios de servicios hoteleros a co-destruir en plataformas hoteleras bajo el mecanismo e-WOM negativo; buscando llenar el vacío de investigación existente. Específicamente, este estudio explorará la relación que tiene la insatisfacción, altruismo, venta de sentimientos negativos, desconfirmación de e-WOM anterior, y desconfianza de e-WOM anterior en la generación de e-WOM negativo, y así establecer las motivaciones que llevan a cierto cliente de servicios hoteleros a co-destruir por medio de e-WOM negativo en las distintas plataformas web.

\section{OTROS ANTECEDENTES}

Hay una serie de antecedentes adicionales que es necesario detallar para documentar en mejor forma este trabajo: i) Co-destrucción de valor; ii) e-WOM negativo como mecanismo de co-destrucción en el turismo hotelero; y iii) Razones para co-destruir a través de e-WOM negativo.

\section{Co-destrucción de valor}

Tal y como describe (Vargo y Lusch, 2016), según la Lógica Dominante del Servicio (LDS), las empresas solo pueden hacer propuestas de valor, ya que el valor se co-crea por medio de las interacciones entre los actores participantes del servicio, incluido el cliente. Durante el proceso de co-creación de valor, la empresa y los clientes aplican sus conocimientos y habilidades, la empresa en la producción y la marca del bien, y el beneficiario en el contexto de su propia vida. De acuerdo con Nam et al. (2020), la aplicación de los supuestos de la LDS al contexto del e-WOM explica porque los consumidores buscan co-crear valor por medio la generación de comentarios o e-WOM sobre sus experiencias tanto con productos como servicios. Por lo tanto, se co-crea valor proporcionando información para otros consumidores, entregando retroalimentación a las empresas, y generando contenido para las plataformas de viajes. Sin embargo, es importante destacar que los recursos que se utilizan positivamente para el beneficio de los sistemas de servicios, también pueden ser utilizados de manera perjudicial para una o todas las partes involucradas, es decir, el cliente puede ser también co-destructor del valor (Plé, 2017). 
La co-destrucción de valor hace referencia a los comportamientos e interacciones donde el intercambio de recursos y actividades entre los actores de servicio conducen a experiencias negativas (Prior y MarcosCuevas, 2016). Según (Plé, 2017), la co-destrucción tiene el potencial de disminuir el bienestar para al menos una de las partes involucradas en el intercambio por alguna discrepancia entre al menos una de las expectativas de los actores que son parte del proceso de integración de recursos. Es importante señalar que la co-creación de valor y la co-destrucción son dos aspectos de formación de valores interactivos que pueden existir simultáneamente; es decir, pueden alternarse entre sí a través del tiempo o incluso coexistir para uno o más actores (Plé, 2017).

\section{e-WOM negativo como mecanismo de co-destrucción en el turismo hotelero}

El e-WOM en forma de reseñas en línea resulta ser de vital importancia para el sector empresarial, ya que es un comportamiento ampliamente utilizado por los consumidores para evaluar productos y servicios, además los dueños de negocios confían en él para construir su reputación (Nam et al., 2020). El e-WOM negativo por su parte, representa a los mensajes de queja en línea contra una empresa los que se distribuyen de una manera rápida y fácil a un gran volumen de consumidores potenciales, esto causa daños a la reputación del negocio, obteniendo menos ingresos debido a los mensajes negativos (Nam et al., 2020).

Dentro del sector turístico, los viajeros utilizan cada vez más las revisiones en línea, para informarse sobre el alojamiento y otros productos relacionados con el turismo. Como tal, es importante mejorar la comprensión de las características del e-WOM (Fernades y Fernandes, 2018). Entre las características útiles y valiosas del e-WOM se encuentran el grado de interacción entre los usuarios, esto por medio de las revisiones, comentarios y calificaciones (Confente, 2015). Los turistas no sólo leen y usan información de internet durante su proceso de elección, sino que también publican información en internet. Así, el receptor obtendrá mayores conocimientos sobre el producto o marca al leer el mensaje, evitando malas elecciones de compra y cambiando sus actitudes previas hacia la marca, por lo que acoger los comentarios del e-WOM negativo es crucial para la empresa.

\section{Razones para co-destruir a través de e-WOM negativo}

Desconfirmación de las Expectativas por e-WOM Previo. La desconfirmación implica una situación en la que el desempeño de un producto o servicio no cumple con las expectativas de los consumidores (Oliver, 1980). Cuando se genera desconfirmación, los consumidores pueden estar motivados por la ira, por la decepción para advertir a los demás, o por el arrepentimiento de fortalecer los vínculos sociales a través de la comunicación con otros consumidores (Nam et al., 2020). Estas tres emociones llevan a los consumidores a expresar sus opiniones acerca de un producto o servicio en respuesta a la desconfirmación de las expectativas, tal como lo señalan (Nam et al., 2020; Sanchez-Garcia y Currás-Pérez, 2011). En base a esto se propone la siguiente hipótesis: H1. La desconfirmación con e-WOM previo tiene un efecto directo y positivo sobre el e-WOM negativo en plataformas hoteleras.

Desconfianza de e-WOM Previo. El e-WOM realizado por otros brinda a los consumidores la oportunidad de recopilar información de productos y revisar evaluaciones en línea. Cuando los consumidores pueden interactuar con los autores del e-WOM, se interesan más en el producto y, por lo tanto, desarrollan una preferencia, aumentando la confianza del consumidor en e-WOM previo (Järvi et al., 2020). Por el contrario, cuando los consumidores han identificado que el e-WOM carece de credibilidad, precisión o confiabilidad según su propia experiencia con un producto o servicio, entonces desconfían del e-WOM previo. En dicho escenario, los consumidores pueden actuar sobre esta desconfianza de múltiples maneras según su propia experiencia. Una de ellas es advertir a otros sobre la buena o mala experiencia que tuvieron con el producto o servicio que se describe en un e-WOM. Esta advertencia puede ser entregada para fortalecer los vínculos sociales con otros consumidores a través de plataformas de revisión en línea con el fin de proporcionar información más verídica del producto o servicio (Nam et al., 2020). Por lo tanto, se plantea la siguiente hipótesis: H2. La desconfianza con el e-WOM previo tiene un efecto directo y positivo con el e-WOM negativo en plataformas hoteleras.

Insatisfacción. La insatisfacción se forma en la mente de un consumidor cuando el desempeño de un producto o servicio no cumple con sus expectativas (Nam et al., 2020). Los clientes insatisfechos pueden optar por cambiar de proveedor de servicio, sin embargo, el e-WOM negativo y las quejas en línea son de los comportamientos más comunes (Sparks y Browning, 2010). Altos niveles de insatisfacción aumentan la probabilidad de generar e-WOM negativo (Fernandes y Fernandes, 2018; Nam et al., 2020). Lo anterior permite determinar la siguiente hipótesis: H3. La insatisfacción con el hotel tiene un efecto directo y positivo con el e-WOM negativo en plataformas hoteleras. 
Venta de Sentimientos Negativos. Cuando los servicios de hotelería y viajes desencadenan sentimientos negativos, los clientes experimentan diferentes emociones, como ira, venganza, frustración y tensión psicológica (Yen y Tang, 2015; Hu y Kim, 2018). La venta de sentimientos negativos hace referencia entonces, al acto de ventilar o descargar los sentimientos negativos, a través de e-WOM negativo. La venta de sentimientos negativos permite a los clientes reducir la ansiedad y frustración, y de este modo restaurar el equilibrio psicológico (Yen y Tang, 2015). Teniendo en cuenta lo anterior se señala la siguiente hipótesis: $H 4$. La venta de sentimientos negativos tiene un efecto directo y positivo sobre el e-WOM negativo en plataformas hoteleras.

Altruismo Negativo. El comportamiento altruista, tiene relación con que un sujeto esté fuertemente motivado tanto por ayudar a otros consumidores como por ayudar a las empresas. Investigadores como Hu y Kim (2018), han señalado que el altruismo es una de las razones que se ha descubierto que explica el e-WOM tanto positivo como negativo. En las experiencias negativas de hospitalidad y viajes, el altruismo significa que los comentarios de e-WOM quieren prevenir que otros experimenten los mismos problemas (Hu y Kim, 2018; Yen y Tang, 2015) y ayudar a la empresa a lograr un mejoramiento (Hu y Kim, 2018). El altruismo en encuentros de servicios negativos, difiere del positivo, que el primero comparte la buena experiencia y apoya a la empresa, mientras que el segundo se enfoca en prevenir a otros de tener una experiencia negativa similar, ayudando a la vez a servir mejor a otros (Hu y Kim, 2018). Así, se desprende la siguiente hipótesis: H5. El altruismo tiene un efecto directo y positivo sobre el e-WOM negativo en plataformas hoteleras. La figura 1 ilustra cada una de las fuentes hipotéticas de un e-WOM negativo las que se detallan a continuación.

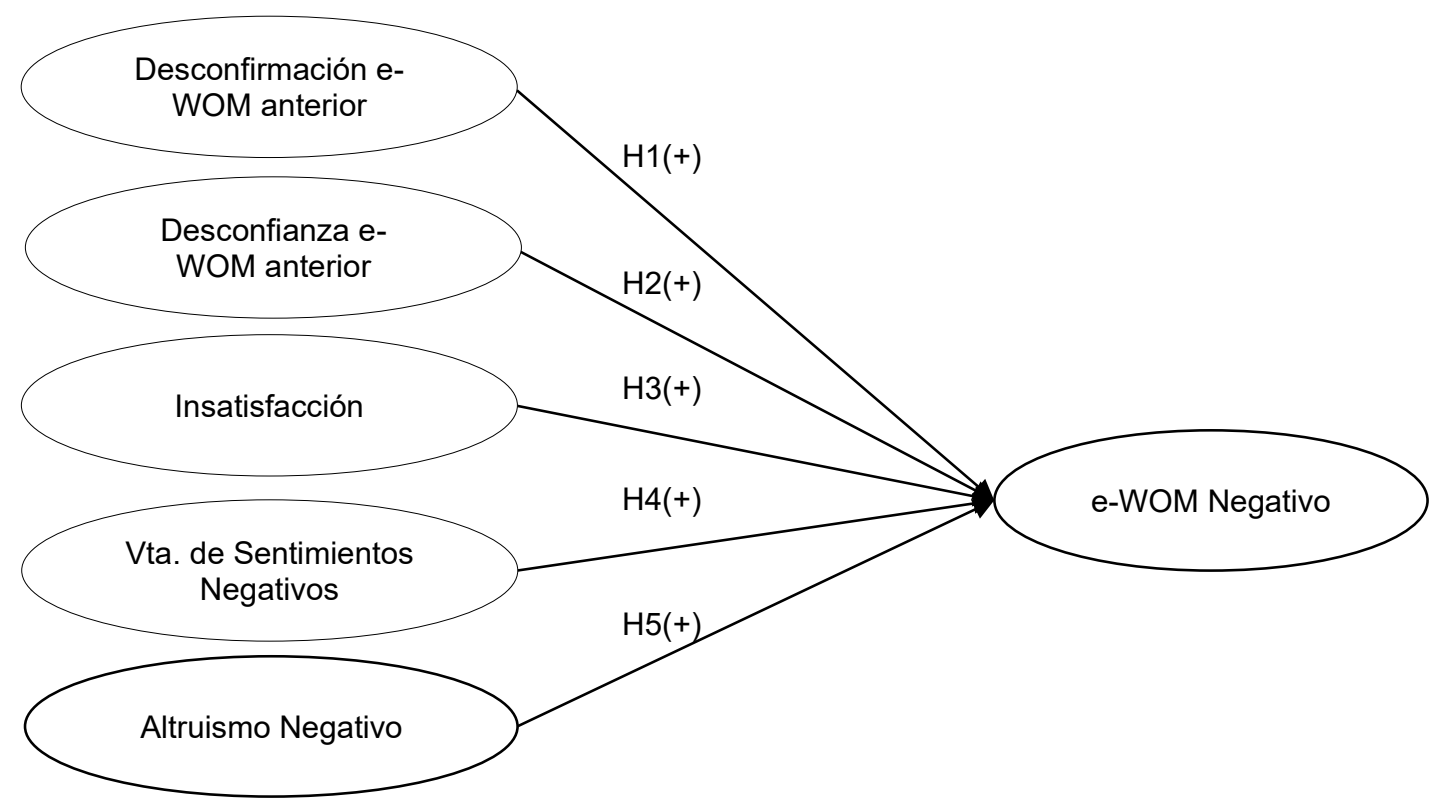

Fig. 1. Variables base de e-WOM negativo.

\section{METODOLOGÍA}

Con el propósito de validar de forma empírica las hipótesis planteadas, se diseño una encuesta estructurda la fue enviada a todos aquellos usuarios de plataformas online hoteleras que visitaron un hotel los últimos 12 meses, y que hayan escrito e-WOM luego de haber adquirido el servicio. El cuestionario fue difundido durante 2 meses usando redes sociales (Facebook, WhatsApp, Instagram) y foros de viajes (TripAdvisor). Mediante cuatro preguntas de filtro, se seleccionaron personas con las siguientes características: (1) Personas mayores de 18 años y menores de 64 años, (2) Si se ha alojado en un hotel u hostal en los últimos doce meses, (3) Que haya tenido una experiencia negativa en su estadía, (4) si ha realizado comentarios o calificaciones negativas posteriores a su experiencia por medio de plataformas virtuales. Todas las personas que no cumplieron con estas características fueron excluidas de la muestra. Un total de 662 respuestas fueron recibidas y 200 de estas fueron usadas para el análisis (ver tabla 1). 
Tabla 1: Cantidad Totales de Respuestas.

\begin{tabular}{|l|l|l|}
\hline Total Encuestas & 662 & \\
\hline Experiencia Negativa & 200 & $30,2 \%$ \\
\hline Experiencia Positiva & 462 & $69,8 \%$ \\
\hline
\end{tabular}

Todas las variables fueron medidas con una escalas de Likert de 5 puntos. Para medir la desconfirmación con el e-WOM previo, la insatisfacción con el hotel y la desconfianza con el e-WOM previo, se utilizó la escala propuesta por (Nam et al., 2020). La venta de sentimientos negativos y el altruismo fue medido a través de la escala propuesta por (Yen y Tang, 2015). Por último, se utilizó la escala propuesta por Boo y Kim (2013) para medir la negatividad del e-WOM . Los indicadores utilizados en este estudio se muestran en la tabla 2. El guión (-) indica las preguntas cuyo puntaje fue revertido. La desconfirmación por e-WOM previo se hizo para captar el grado con que los encuestados, a través de su experiencia, confirmaron o no las reseñas previamente leídas. Luego el puntaje fue revertido.

Tabla 2: Elementos de Medición.

\begin{tabular}{|c|c|c|}
\hline Variable & Indicador & Fuente \\
\hline $\begin{array}{l}\text { Desconfirmación } \\
\text { con el e-WOM } \\
\text { previo }\end{array}$ & $\begin{array}{l}\text { Mi experiencia con el hotel fue tan buena como las reseñas previamente } \\
\text { leídas (-). } \\
\text { El servicio que brindaba el hotel fue tan bueno como las reseñas } \\
\text { previamente leídas (-). } \\
\text { En general, la mayoría de mis expectativas con el hotel se cumplieron } \\
\text { como fue descrito en las reseñas leídas previamente (-) }\end{array}$ & Nam et al. (2020) \\
\hline $\begin{array}{l}\text { Insatisfacción } \\
\text { con el hotel }\end{array}$ & $\begin{array}{l}\text { Tu experiencia en general con el hotel fue: } \\
\text { (Insatisfactoria-Satisfactoria)(-) } \\
\text { (Muy frustrante-Muy placentera)(-) }\end{array}$ & Nam et al. (2020) \\
\hline $\begin{array}{l}\text { Desconfianza } \\
\text { del e-WOM } \\
\text { previo }\end{array}$ & $\begin{array}{l}\text { Creo que las revisiones anteriores son... } \\
\text { No son creíbles. (Muy en desacuerdo-Muy de acuerdo) } \\
\text { Son inexactas. (Muy en desacuerdo-Muy de acuerdo) } \\
\text { No son confiables. (Muy en desacuerdo-Muy de acuerdo) }\end{array}$ & Nam et al. (2020) \\
\hline $\begin{array}{l}\text { Venta de } \\
\text { sentimientos } \\
\text { negativos }\end{array}$ & $\begin{array}{l}\text { Prefiero dar a conocer mi angustia sobre la mala experiencia que viví } \\
\text { Prefiero desahogar mis sentimientos negativos sobre la experiencia } \\
\text { Un servicio tan malo como el que experimente debería ser sancionado }\end{array}$ & Yen y Tang (2015) \\
\hline Altruismo & $\begin{array}{l}\text { Me gustaría advertirles a otros para evitar que tengan una mala } \\
\text { experiencia como la mía } \\
\text { Me gustaría ayudar al hotel a mejorar en sus servicios }\end{array}$ & Yen y Tang (2015) \\
\hline e-WOM negativo & $\begin{array}{l}\text { Comparto la información online con otros para evitarles que tomen una } \\
\text { decisión riesgosa } \\
\text { Comparto la información online con otros para poder contarle sobre mi } \\
\text { experiencia negativa } \\
\text { Emitir comentarios online es la manera más fácil de compartir mi } \\
\text { experiencia negativa en el hotel }\end{array}$ & Boo y Kim (2013) \\
\hline
\end{tabular}

\section{RESULTADOS}

Un $35,5 \%$ de los encuestados se identifica como masculino y el $64,5 \%$ como femenino. Como muestra la tabla 3 , el rango de edad de los encuestados era entre 18-25 años. A partir de los resultados obtenidos es posible apreciar que el $91 \%$ de los encuestados si buscó información antes de experimentar el servicio, donde el $78,5 \%$ emitió alguna queja o sugerencia en el hospedaje. Además, cabe destacar, que la plataforma web donde más comentarios se realizaron fue Booking con un $51 \%$ y lo sigue Facebook con un $17 \%$. El resumen con el total de datos obtenidos se presenta a continuación en la tabla 4. Se utilizó el método de mínimos cuadrados parciales (PLS) con el programa SMARTPLS 3.2.7 (Ringle et al., 2014) para realizar una evaluación simultánea de la calidad de la medición y de la validez de las hipótesis. La técnica PLS es apropiada para este estudio, ya que está orientada a la predicción, por lo que es adecuada para evaluar teorías en etapas de desarrollo inicial. Además, se utilizó la técnica de cálculos Bootstrapping para obtener información más detallada acerca del estudio, para evaluar el significado de los coeficientes de ruta. 
Tabla 3: Características Demográficas

\begin{tabular}{|l|c|c|c|}
\hline Características & Categoría & Frecuencia & $\%$ \\
\hline Sexo & Femenino & 129 & $64,5 \%$ \\
& Masculino & 71 & $35,5 \%$ \\
\hline Edad & $18-25$ & 106 & $53 \%$ \\
& $26-34$ & 61 & $30,5 \%$ \\
& $35-44$ & 21 & $10,5 \%$ \\
& $45-54$ & 11 & $5,5 \%$ \\
& $55-64$ & 1 & $0,5 \%$ \\
\hline
\end{tabular}

Tabla 4: Preguntas Análisis Descriptivo

\begin{tabular}{|c|c|c|c|}
\hline Preguntas & Categoría & Frecuencia & $\%$ \\
\hline $\begin{array}{l}\text { ¿Buscó información por medio de plataformas online } \\
\text { antes de adquirir el servicio? }\end{array}$ & $\begin{array}{l}\mathrm{Si} \\
\mathrm{No}\end{array}$ & $\begin{array}{c}183 \\
17\end{array}$ & $\begin{array}{l}91,5 \% \\
8,5 \%\end{array}$ \\
\hline $\begin{array}{l}\text { ¿Ha empleado comentarios o calificaciones negativas } \\
\text { posteriores a su experiencia por medio de plataformas } \\
\text { virtuales? }\end{array}$ & $\begin{array}{l}\text { Si } \\
\text { No }\end{array}$ & $\begin{array}{c}157 \\
43\end{array}$ & $\begin{array}{l}78,5 \% \\
21,5 \%\end{array}$ \\
\hline $\begin{array}{l}\text { En relación a la emisión de comentarios negativos } \\
\text { online, ¿En qué plataforma lo realizó? }\end{array}$ & $\begin{array}{l}\text { Booking } \\
\text { Tripadvisor } \\
\text { Trivago } \\
\text { Facebook } \\
\text { Twitter } \\
\text { Otra }\end{array}$ & $\begin{array}{c}102 \\
26 \\
10 \\
34 \\
6 \\
22\end{array}$ & $\begin{array}{c}51 \% \\
13 \% \\
5 \% \\
17 \% \\
3 \% \\
11 \%\end{array}$ \\
\hline $\begin{array}{l}\text { ¿Con qué propósito efectuó comentarios negativos en } \\
\text { la plataforma online? }\end{array}$ & $\begin{array}{l}\text { Advertir a futuros consumidores } \\
\text { Compensación monetaria } \\
\text { Compensación no monetaria } \\
\text { Recomendación para el } \\
\text { proveedor del servicio } \\
\text { Otra }\end{array}$ & $\begin{array}{c}132 \\
10 \\
3 \\
52 \\
3\end{array}$ & $\begin{array}{c}66 \% \\
5 \% \\
1,5 \% \\
26 \% \\
1,5 \%\end{array}$ \\
\hline Motivo del Viaje & $\begin{array}{l}\text {-Negocios, Trabajo } \\
\text {-Recreación, Vacaciones } \\
\text {-Visitas a familiares y/o amigos } \\
\text {-Asistencia Eventos } \\
\text {-Otros Motivos }\end{array}$ & $\begin{array}{c}25 \\
147 \\
10 \\
10 \\
8\end{array}$ & $\begin{array}{c}12,5 \% \\
73,5 \% \\
5 \% \\
5 \% \\
4 \%\end{array}$ \\
\hline
\end{tabular}

El modelo propuesto (ver figura 2) es de carácter reflexivo, por ende fue evaluado respecto a su fiabilidad y validez. La evaluación de fiabilidad del modelo se enfoca en la fiabilidad compuesta como una estimación de la consistencia interna de una construcción (Hair et al., 2011). Este modelo fue evaluado a través de pruebas de fiabilidad, validez convergente, validez discriminante, consistencia interna (fiabilidad compuesta). Para verificar la validez convergente, se evaluó la varianza extraída (AVE) de cada variable latente, donde el umbral aceptable es de al menos de 0.5 (Fornell y Larcker, 1981), la fiabilidad compuesta (FC) con un mínimo de 0.7 (Nunnally y Bernstein, 1994) y las cargas cruzadas mayores a 0.7 (Carmines y Zeller, 1979). En este caso las tres condiciones se cumplen, confirmando la validez convergente, en el que AVE obtuvo rangos entre [0.6, $0.9]$ y la FC entre $[0.7,0.9]$ (Ver tabla 5).

Los resultados obtenidos indicaron que el modelo de medida confirma la validez convergente, pese que dos indicadores presenten una leve alteración en las cargas cruzadas, los cuales se decidió mantener y con esto asegurar la validez de contenido de las escalas. Cabe destacar que, a diferencia del alfa de Cronbach, la fiabilidad compuesta (FC) no supone que todos los indicadores sean igualmente confiables, lo que es más adecuado para PLS-SEM, ya que prioriza los indicadores según su fiabilidad durante la estimación del modelo (Hair et al., 2011).

La validez discriminante indica en qué medida un constructo determinado es diferente de otros constructos (Martínez Ávila Y Fierro Moreno, 2018). Para valorar la validez discriminante, es necesario evaluar el criterio de Fornell-Lacker (Fornell y Larcker 1981), el cual considera la cantidad de varianza que un constructo captura de sus indicadores (AVE), el cual debe ser mayor a la varianza que el constructo comparte con otros constructos. En este, la raíz cuadrada de AVE de cada variable latente deberá ser mayor que las correlaciones que tiene con el resto de las variables, es decir, mayor que la correlación que este tenga con cualquier constructo. Según los resultados que se encuentran en la tabla 6 , todos los coeficientes de cada constructo se encuentran en un rango de $[0.811,0.973]$ y son mayores a los obtenidos con el resto de los constructos. Esto significa que el criterio de Fornell-Lacker se cumple para la validez discriminante. Con respecto al modelo 
estructural y la contrastación de hipótesis, se utilizó en primer lugar como criterio principal para la evaluación del modelo estructural es la cantidad de la varianza explicada $R^{2}$ (Chin, 1998). En la figura 2 , se aprecia un valor $\mathrm{R}^{2}$ de la variable e-WOM negativo de 0.414 , lo cual indica que las variables independientes del modelo planteadas como razones para co-drestruir, explican en un 41,4\% la forma de co-drestrucción e-WOM negativo. Además se puede ver que los coeficientes path entre constructos, cumplen con el valor mínimo 0,2 (Chin, 1998). Por último, para el contraste de hipótesis se aplicó la técnica de PLS boostraping utilizando 5000 muestras (Streukens y Leroi-Werelds, 2016) y se calculó los path loadings y los P-valor para las relaciones descritas en las hipótesis. Los resultados se muestran en la tabla 7.

Tabla 5: Resumen de los Resultados

\begin{tabular}{|l|l|l|l|l|}
\hline Variable Latente & \multicolumn{1}{|c|}{ Indicador } & Cargas & FC & AVE \\
\hline Altruismo & Altruismo1 & 0.942 & 0.788 & 0.658 \\
& Altruismo2 & 0.654 & & \\
\hline Desconfianza & Desconfianza1 & 0.622 & 0.862 & 0.682 \\
& Desconfianza2 & 0.882 & & \\
& Desconfianza3 & 0.939 & & \\
\hline Desconfirmación & Desconfirmación1 & 0.904 & 0.931 & 0.817 \\
& Desconfirmación2 & 0.924 & & \\
& Desconfirmación3 & 0.883 & & \\
\hline e-WOM negativo & e-WOM1 & 0.874 & 0.879 & 0.710 \\
& e-WOM 2 & 0.912 & & \\
& e-WOM 3 & 0.732 & & \\
\hline Insatisfacción & Insatisfacción1 & 0.965 & 0.973 & 0.948 \\
& Insatisfacción2 & 0.981 & & \\
\hline Vta.sentimientos & Vta.sentimientos1 & 0.732 & 0.862 & 0.676 \\
& Vta.sentimientos2 & 0.883 & & \\
& Vta.sentimientos3 & 0.945 & & \\
\hline
\end{tabular}

Tabla 6: Criterio de Fornell-Larcker.

\begin{tabular}{|l|l|l|l|l|l|l|}
\hline $\begin{array}{l}\text { Validez } \\
\text { Discriminante }\end{array}$ & Altruismo & Desconfianza & Desconfirmación & $\begin{array}{l}\text { e-Wom } \\
\text { Negativo }\end{array}$ & Insatisfacción & $\begin{array}{l}\text { Vta. } \\
\text { Sentimientos }\end{array}$ \\
\hline Altruismo & 0.811 & & & & & \\
\hline Desconfianza & 0.020 & 0.826 & & & & \\
\hline Desconfirmación & 0.087 & 0.159 & 0.904 & & & \\
\hline e-Wom Negativo & 0.587 & 0.029 & 0.182 & 0.843 & & \\
\hline Insatisfacción & 0.134 & 0.062 & 0.790 & 0.280 & 0.973 & \\
\hline Vta.Sent. Negativos & 0.590 & 0.130 & 0.281 & 0.521 & 0.352 & 0.822 \\
\hline
\end{tabular}

Tabla 7: Coeficientes Path (Bootstrapping)

\begin{tabular}{|c|c|c|c|c|}
\hline Coeficientes Path & $\begin{array}{l}\text { Muestra } \\
\text { original }\end{array}$ & $\begin{array}{c}\text { Estadísticos } \\
t\end{array}$ & $\begin{array}{c}P \\
\text { Valores }\end{array}$ & $\begin{array}{c}\text { Resultado } \\
{ }^{*} \text { Significancia } P<0.05\end{array}$ \\
\hline Altruismo -> e-WOM Negativo & 0.443 & 6.155 & 0.000 & Soportada * \\
\hline Desconfianza -> e-WOM Negativo & -0.007 & 0.095 & 0.924 & No soportada* \\
\hline Desconfirmación -> e-WOM Negativo & -0.082 & 0.938 & 0.348 & No soportada* \\
\hline Insatisfacción -> e-WOM Negativo & 0.213 & 2.427 & 0.015 & Soportada* \\
\hline Vta. Sentimientos Negativos -> e-WOM Negativo & 0.209 & 2.261 & 0.024 & Soportada* \\
\hline
\end{tabular}




\section{DISCUSIÓN}

La co-destrucción, al ser un elemento de investigación nuevo en el área de los servicios, representa un escenario donde una gran cantidad de personas no ejerce este tipo de acciones. Las calificaciones hacia el servicio hotelero son la base para conocer en profundidad los aspectos positivos y negativos del mismo, y son de gran ayuda para los proveedores de servicio hoteleros para conocer las causas de comportamiento co-destructivo hacía el servicio. Los resultados de esta investigación revelan que las personas que codestruyen por medio de e-WOM negativo, lo hacen movidos por la insatisfacción con el servicio recibido tal como lo señalan (Fernandes y Fernandes, 2018; Nam et al., 2020), además con la idea de ayudar a otros a no pasar por la misma experiencia que ellos lo que tiene relación con el altruismo que busca prevenir que otros experimenten los mismos problemas (Hu y Kim, 2018; Yen y Tang, 2015); y la venta de sentimientos negativos (Yen y Tang, 2015). Dos variables de este estudio no presentaron un efecto significativo en la codestrucción por medio del e-WOM los cuales son la desconfianza en el e-WOM anterior y la desconfirmación con el e-WOM anterior, lo anterior puede deberse a que la experiencia real del cliente con el servicio tenga más peso que los comentarios, que este allá podido leer anteriormente.

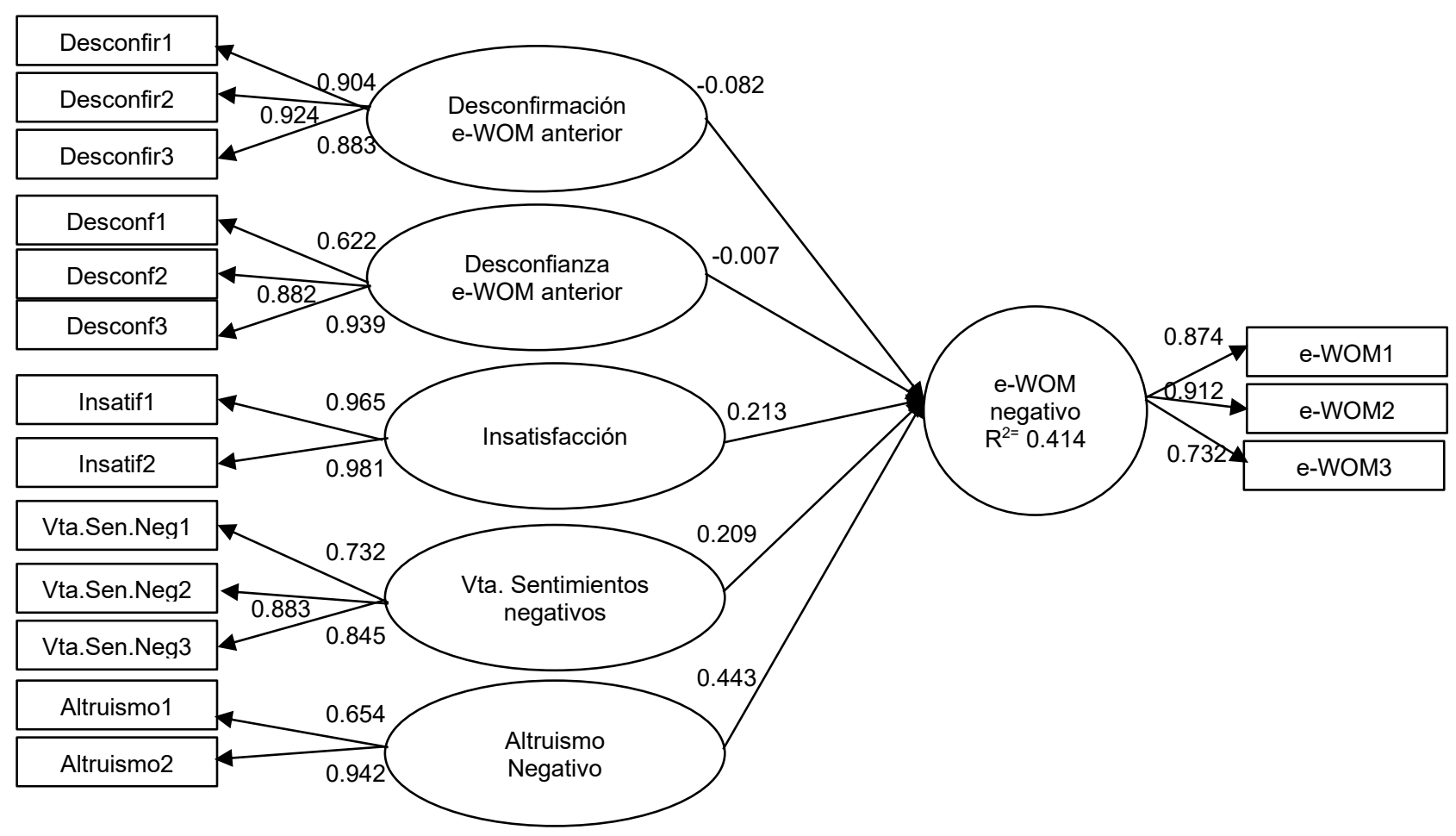

Fig. 2. Modelo en Función SmartPLS.

Conocidas las razones o motivaciones para co-destruir, los proveedores de servicios hoteleros, deben preocuparse de satisfacer las necesidades de sus clientes, puesto que, ellos al no estar vinculados a una página web, procuran obtener el servicio por el cual se formularon una expectativa. Los servicios hoteleros deben cumplir lo ofrecido, ya que, si bien no hay una relación negativa a la plataforma de revisión, si puede estarlo a los servicios que brindan y relacionarlo directamente al hotel.

Para futuras investigaciones, se debiese considerar respuestas de encuestas en formato tradicional (presencial) en sectores claves tales como hoteles, hostales, entre otros, para así comparar ambos resultados y validar los resultados de las encuestas en línea. También se considera que un estudio futuro también con límites más amplios de edad, debido a que fue posible corroborar que personas de más avanzada edad también emiten e-WOM negativo. Por último, la unidad de muestreo no solo considera las personas que han viajado los últimos doce meses, si no a todas aquellas que han tenido una mala experiencia en el rubro seleccionado, para así medir de mejor forma el comportamiento co-destructivo. Se esperar también considerar otros rubros en los cuales hay co-destrucción, por ejemplo, los restaurantes. Se espera que este estudio sea un aporte para futuros investigadores de la co-destrucción del valor. Los resultados obtenidos podrán contribuir en la profundización del tema, con el objetivo de que se incluyan nuevos aspectos a futuro y que este sea fundamental para lograr un análisis más completo y reconocido. 


\section{CONCLUSIONES}

De acuerdo al trabajo presentado y a los resultados obtenidos, se pueden plantear las siguientes conclusiones principales:

1.- Las personas que co-destruyen por medio de e-WOM negativo realizan esta acción por la insatisfacción con el servicio recibido

2.- Las personas que co-destruyen por medio de e-WOM negativo realizan esta acción con la idea altruista de ayudar a otros para que no vivan la misma experiencia negativa.

3.- Tanto la desconfianza como la desconfirmación de e-WOMs previos no representan variables que impacten en la consideración de comentarios negativos ya leidos.

\section{REFERENCIAS}

Boo, S., y Kim, J., Comparison of negative eWOM intention: an exploratory study, http://doi.org/10.1080/1528008X.2013.749381, J. Qual. Assur. Hosp. Tour., 14(1), 24-48 (2013)

Booking, Booking.com, (en línea), https://www.booking.com/index.html, acceso: 06 de Septiembre (2020)

Busser, J., y Shulga, L., Co-created value: multidimensional scale and nomological network, http://doi.org/10.1016/j.tourman.2017.09.014, Tourism Management, 65, 69-86 (2018)

Carmines, E. y Zeller, R., Quantitative applications in the social sciences: reliability and validity assessment, http://doi.org/10.4135/9781412985642, Thousand Oaks, CA: SAGE Publications, Inc. (1979)

Casais, B., Fernandes, J., y Sarmento, M., Tourism innovation through relationship marketing and value co-creation: a study on peer-to-peer online platforms for sharing accommodation, http://doi.org/10.1016/j.jhtm.2019.11.010, Journal of Hospitality and Tourism Management, 42, 51-57 (2020)

Confente, I., Twenty-five years of word-of-mouth studies: a critical review of tourism research, http://doi.org/10.1002/jtr.2029, International Journal of Tourism Research, 17(6), 613-624 (2015)

Fan, D., Hsu, C., y Lin, B., Tourists' experiential value co-creation through online social contacts: customer-dominant logic perspective, http://doi.org/10.1016/j.jbusres.2019.11.008, Journal of Business Research, 108, 163-173 (2020)

Fernandes, T. y Fernandes, F., Sharing dissatisfaction online: analyzing the nature and predictors of hotel guests negative reviews, http://doi.org/10.1080/19368623.2017.1337540, Journal of Hospitality Marketing \& Management, 27(2), 127-150 (2018)

Fornell, C. y Larcker, D., Evaluating structural equation models with unobservable variables and measurement error, http://doi.org/10.1177/002224378101800104, Journal of Marketing Research, 18, $39-50$ (1981)

Gonçalves, H., Silva, G., y Martins, T., Motivations for posting online reviews in the hotel industry, http://doi.org/10.1002/mar.21136, J. Psychology \& Marketing, 35(11), 807-817 (2018)

Hair, J., Ringle, C., y Sarstedt, M., PLS-SEM: indeed a silver bullet, http://doi.org/10.2753/MTP1069-6679190202, Journal of Marketing Theory and Practice, 19(2), 139-152 (2011)

$\mathrm{Hu}, \mathrm{Y}$. y Kim, H., Positive and negative eWOM motivations and hotel customers' eWOM behavior: does personality matter?, http://doi.org/10.1016/j.jijhm.2018.03.004, International Journal of Hospitality Management, 75, 27- 37 (2018)

Järvi, H., Keränen, J., Ritala, P., y Vilko, J., Value co-destruction in hotel services: exploring the misalignment of cognitive scripts among customers and providers, http://doi.org/10.1016/j.tourman.2019.104030, Tourism Management, 77, 104030 (2020)

Kashif, M., y Zarkada, A., Value co-destruction between customers and frontline employees, http://doi.org/10.1108/IJBM09-2014-0121, International Journal of Bank Marketing, 33(6), 672-691 (2015)

Martínez Ávila, M., y Fierro Moreno, E., Aplicación de la técnica PLS-SEM en la gestión del conocimiento: un enfoque técnico práctico, http://doi.org/10.23913/ride.v8i16.336, RIDE Revista Iberoamericana para la Investigación y el Desarrollo Educativo, vol. 8 (2018)

Media, Preferente, (en línea), https://www.preferente.com/noticias-de-hoteles/los-hoteles-dependen-cada-vez-mas-delas-plataformas-online-278546.html, acceso: 05 de Septiembre (2020)

Nam, K., Baker, J., Ahmad, N., y otros 2 autores, Dissatisfaction, disconfirmation, and distrust: an empirical examination of value co-destruction through negative electronic word-of-mouth (eWOM), http://doi.org/10.1007/s10796-018-9849-4, Information System Frontiers, 22(1), 113-130 (2020)

Nunnally, J., y Bernstein, I., Psychometric theory, http://doi.org/10.1177/014662169501900308, 3era Edición, McGrawHill, 19(3), 303-305 (1994)

Oliver, R., A cognitive model of the antecedents and consequences of satisfaction

decisions, http://doi.org/10.1177/002224378001700405, Journal of Marketing Research, 17(4), 460-469 (1980) 
Plé, L., Why do we need research on value co-destruction?, http://doi.org/10.1177/2394964317726451, Journal of Creating Value, 9, 239496431772645 (2017)

Prebensen, N., y Xie, J., Efficacy of co-creation and mastering on perceived value and satisfaction in tourists' consumption, http://doi.org/10.1016/j.tourman.2016.12.001, Tourism Management, 60, 166-176 (2017)

Prior, D., y Marcos-Cuevas, J., Value co-destruction in interfirm relationships: the impact of actor engagement styles, http://doi.org/10.1177/1470593116649792, Marketing Theory, 16(4), 533-552 (2016)

Ringle, C., Wende, S., y Becker, J., SmartPLS 3, Bönningstedt: SmartPLS Gmbh, 10-120 (2014)

Sparks, B., y Browning, V., Complaining in cyberspace: the motives and forms of hotel guests' complaints online, http://doi.org/10.1080/19368623.2010.508010, Journal of Hospitality Marketing \& Management, 19(7), $797-818$ (2010)

Streukens, S. y Leroi-Werelds, S., Bootstrapping and PLS-SEM: a step-by-step guide to get more out of your bootstrap results, http://doi.org/10.1016/j.emj.2016.06.003, European Management Journal, 34(6), 618-632 (2016)

Ukeje, U., Lasisi, T., Eluwole, K., y otros 2 autores, Organizational level antecedents of value co-destruction in hospitality industry: an investigation of the moderating role of employee attribution, http://doi.org/10.1080/13683500.2020.1763928, Current Issues in Tourism, 1-15 (2020)

Vargo, S., y Lusch, R., Institutions and axioms: an extension and update of service-dominant logic, http://doi.org/10.1007/s11747-015-0456-3, Journal of the Academy of Marketing Science, 44(1), 5-23 (2016)

Worthington, S., y Durkin, M., Co-destruction of value in context: cases from retail banking, http://doi.org/10.1362/146934712X13420906885476, The Marketing Review, 12(3), 291-307 (2012)

Yen, C., y Tang, C., Hotel attribute performance, eWOM motivations, and media choice,

http://doi.org/10.1016/j.ijhm.2015.01.003, International Journal of Hospitality Management, 46, 79-88 (2015) 